\title{
Construcción social de los problemas conductuales en infantes
}

\section{Nayeli Arechiga' y Felipe Díaz ${ }^{2}$ \\ Laboratorio de Análisis Conductual, Centro Universitario del Sur - Universidad de Guadalajara}

\section{Ensayo}

Material original autorizado para su primera publicación en Journal de Ciencias Sociales, Revista Académica de la Facultad de Ciencias Sociales de la Universidad de Palermo.

Recepción: 25-11-2020

Aceptación: 10-03-2021

Resumen: Los problemas de conducta durante la infancia tienen afectaciones importantes tanto en quienes los presentan como en las personas que interactúan frecuentemente con ellos. Estas afectaciones son más evidentes en los contextos familiares y educativos que durante los últimos meses han sido seriamente modificados debido a la pandemia causada por COVID-19, así como sus repercusiones sociales, políticas y económicas. Más que en otros momentos, referirse a los problemas conductuales infantiles cobra importancia a nivel mundial. La experiencia que están pasando las familias con niños en primera infancia repercutirá en el futuro inmediato y su impacto se reflejará en las áreas del dominio psicológico y social. Aunado a esta predicción está el hecho de que la atención adecuada para estos problemas sigue siendo un reto, sobre todo, para países en vías de desarrollo como México. Existen elementos sociales y estructurales que pueden estar dificultando la atención temprana e intensiva hacia los niños y niñas. En el presente ensayo se discutirá acerca de la construcción social de los problemas conductuales infantiles, así como la forma en que dicha construcción es influenciada por elementos sociales y estructurales. Al principio del documento se presentan algunos datos estadísticos que permiten al lector dimensionar la naturaleza del problema. Posteriormente se argumenta sobre la importancia de contextualizar la conducta objetivo y se plantean algunos ejemplos cotidianos que buscan facilitar la reflexión. Enseguida se expone una problemática adyacente a los problemas

\footnotetext{
${ }^{1}$ Licenciada en Psicología por la Universidad de Guadalajara. Maestra en Psicología por la Universidad Autónoma de San Luis Potosí. Estudiante de Doctorado en Psicología con Orientación en Calidad de Vida y Salud por la Universidad de Guadalajara.

Correo electrónico: nayeliarechiga@gmail.com

2 Doctor en Psicología por la UNAM. Dedicado al Análisis Experimental del Comportamiento. Profesor-Investigador Titular, A, TC. Centro Universitario del Sur, Universidad de Guadalajara. Correo electrónico: felipe.resendiz@cusur.udg.mx
} 
conductuales de los infantes: el desgaste del cuidador. Se finaliza el ensayo con la urgencia de dirigir esfuerzos hacia la puesta en práctica de formas de intervención con respaldo empírico como lo es el análisis conductual aplicado.

Palabras clave: problemas conductuales; contexto; cuidadores; infancia.

\section{Children's behavior problems social construction}

Abstract: Childhood behavior problems have important effects on children and on the people in touch with them. These effects are more evident in family and educational contexts, which in recent months, have been seriously affected due to the pandemic caused by COVID-19, as well as the related social, political and economic repercussions. More than other times, talking about childhood behavioral problems have importance worldwide. Current experiences of families with early childhood kids will have repercussions on immediate future and its impact will be seen in areas of social and psychological domain. Coupled with this prediction it is the fact that appropriate attention to these problems remain a challenge, specially to developing countries like Mexico. There are social and structural elements that may be hampering early and intensive care for children. This essay will discuss about the social and structural elements that influence the treatment of children's behavior problems. At the beginning of the document some statistical data are presented to allow the reader to assess the dimension of the problem. Subsequently, with the objective of facilitate a reflective positioning, some arguments are provided about the importance of contextualizing behavior problems and some examples are raised to this purpose. Next, an adjacent problem is exposed: the burnout in caregivers and the emerging telehealth treatment for the families. This essay concludes pointing out the urgency of directing efforts towards putting into practice interventions with empirical support such as applied behavior analysis.

Keywords: behavior problems; context; caregivers; childhood.

\section{Introducción}

Un importante cuerpo de literatura científica relaciona las conductas disruptivas en la infancia con la aparición de dificultades académicas, sociales, comportamiento delictivo, alteraciones psicológicas y trastornos psiquiátricos (Boisjoli et al. 2007; Fischer et al. 2019; Hagan-Burke et al. 2011; Hodgings et al. 2013; Tremblay, 2004; Tremblay y Côté, 2019; Vitaro et al. 2005). Las conductas disruptivas son el eje central de los conflictos que presenta la población infantil (Valencia y Andrade, 2005) y constituyen uno de los motivos más frecuentes de consulta y asistencia psicológica, neurológica y psiquiátrica (De la Peña-Olvera y PalaciosCruz, 2011). En los casos en que estas conductas no se atienden, no desaparecen con el 
paso del tiempo o por un efecto de maduración biológica, de hecho, el riesgo de que se conviertan en el inicio de un estilo de vida delincuencial es alto.

Las consecuencias de dichas alteraciones pueden afectar seriamente la calidad de vida, no solamente de los niños, niñas y adolescentes, sino también de todas las personas que interactúan con ellos. El estudio de estas afectaciones debe realizarse de manera cuidadosa con la finalidad de no estigmatizar los problemas de conducta que presentan las personas. Tonon (2007) señaló que a pesar de que existen diversas conceptualizaciones sobre la calidad de vida, los estudios más recientes muestran una inclinación hacia una mirada desde las potencialidades más que desde las carencias. En este sentido, la individualidad de las personas toma especial importancia para la toma de decisiones en el tratamiento de las conductas disruptivas. De esta manera se promueve una visión donde la deficiencia o el diagnóstico psiquiátrico deja de ser el centro de atención y se da paso a una concepción centrada en la persona y en su potencial.

Pero ¿qué es un problema conductual? En el ámbito científico, específicamente del análisis de la conducta, existen diferentes denominaciones para referirse a los problemas de conducta tales como comportamientos disruptivos, no deseados, desadaptativos, disfuncionales, entre otras. Si se parte de la premisa de que la relevancia social de la literatura científica se pone a prueba cuando se valida su utilidad en la vida cotidiana, será evidente que más allá de estas conceptualizaciones, son las personas, en sus comunidades, quienes construyen estos conceptos con base en sus propias experiencias.

Es necesario entonces situarse en un momento y lugar específico puesto que todo comportamiento responde a interacciones específicas $y$, como tal, no es posible definirlo fuera de su contexto. Cabe aclarar que, siempre es desde la mirada de otro que estas conductas son juzgadas, valoradas o catalogadas como disruptivas y que esta valoración depende del contexto en el que se emiten. Así, un sujeto inmerso en un contexto donde delinquir es parte de lo que se espera para integrarse a un grupo o que la condición de vida está permeada por una esfera social económicamente desfavorecida, dichas conductas no son consideradas disruptivas sino apropiadas al contexto. Es necesario precisar que, a pesar de la condición desfavorecida en la que se pueda pensar, las normas sociales, la legislación y la actividad productiva son un bien común por encima de los beneficios particulares de una parte de la sociedad. Por lo que, es un objetivo prioritario incorporar a los individuos a conducirse siempre que sea posible, bajo las normas sociales de convivencia que contribuyen con la convivencia pacífica y solidaria entre los ciudadanos del mundo (UNESCO, 2020).

El propósito del presente ensayo es discutir sobre la construcción social de los problemas conductuales infantiles, así como la forma en que dicha construcción es 
influenciada por elementos sociales y estructurales y las implicaciones en torno a las posibilidades de tratamiento.

\section{Argumentación teórica}

\subsection{La contextualización como criterio indispensable}

Investigaciones en el área estiman que entre el 7 y $34 \%$ de los estudiantes de preescolar presentan problemas de conducta de alta intensidad y que este porcentaje parece mantenerse en los grados de educación primaria y en la vida posterior (Benjet et al. 2010; Fischer et al. 2019; Fombonne et al. 2016). Pero esto es solo el principio. Existe evidencia de que cuando los problemas de conducta infantiles no se tratan a tiempo, éstos además de permanecer, tienden a aumentar en frecuencia y severidad hacia la vida adulta.

Si las repercusiones son tan relevantes para la vida de las personas ¿cuál es el motivo por el que no se atienden a tiempo? Existen razones de índole social, estructural, económica e idiosincrática. Revisar estas razones es parte medular del presente ensayo puesto que justamente son elementos que influyen en la forma en que se perciben los problemas conductuales en cada sociedad.

En el caso de México, el análisis de las causas tendrá que comenzar por las razones estructurales y económicas, pues para acudir a instituciones que evalúan, diagnostican y tratan problemas de conducta, es requisito indispensable que estas instituciones existan, que dispongan de la infraestructura necesaria, posean personal capacitado y que la ciudadanía tenga acceso a estos servicios. En México, menos del 25\% de las unidades médicas cuentan con por lo menos un profesional capacitado para brindar atención a niños con trastornos del espectro autista, por lo que la disponibilidad de servicios sigue siendo una importante área de oportunidad (Zavaleta et al. 2018). Adicionalmente, la ley en México que respalda este tipo de intervenciones es relativamente reciente y a la fecha no ha tenido el impacto positivo sobre la detección, diagnóstico, tratamiento y seguimiento del problema. Permea a esta problemática un ambiente político en el que cada nueva administración desestima las propuestas de la anterior. Un contexto estructural que mantiene en el anonimato y, por tanto, fuera del alcance de cualquier tratamiento del sistema de salud mexicano a un número desconocido de niñas y niños de todo el territorio nacional.

En lo que respecta a las causas sociales, resalta que por lo general los casos de niños que requieren algún tipo de atención terapéutica son detectados de manera tardía, cuando las ganancias comportamentales son menores en comparación con una intervención temprana e intensiva. En los primeros años de vida existen mayores oportunidades de modificar las conductas de los niños (Fombonne et al. 2016). Adicionalmente, se ha visto que 
no se presta suficiente atención a los problemas de conducta hasta que el problema es más severo o afecta a mayor cantidad de personas (Tremblay y Côté, 2019). Probablemente, la ayuda se solicitará una vez que el sujeto ya ha delinquido o en casos menos severos cuando se recibió un reporte de comportamiento inapropiado en un contexto escolar.

Lo antes expuesto pone de manifiesto que la construcción social que se hace de los problemas conductuales tiene que ver con la misma visibilidad que se le da a la cuestión, con la forma en que cada familia decide si una conducta determinada le genera inconvenientes. Adicionalmente, es posible que si las personas no reciben información y no tienen acceso a instituciones que los orienten en el tratamiento de estos problemas, pueden creer que no hay nada que hacer al respecto, se vuelve cotidiano convivir con el problema e, incluso, no se percibe como tal.

Ahora bien, la conceptualización que se hace de los problemas conductuales no es del todo consensuada, incluso dentro de la psicología. Para una mejor comprensión del comportamiento y evitar errores categoriales, pensemos en un caso. Sofía es una niña de cinco años de edad que frecuentemente rechaza el alimento que sus padres le dan en casa; generalmente dice que no le gusta la comida, la tira sobre la mesa o el piso y lo padres no logran hacer que su hija consuma el alimento. Después de un largo rato de insistencia, Sofía comienza a llorar y a decirle a sus padres que son muy malos con ella, lanza los utensilios de comida y cada vez que intentan darle un bocado, ella lo rechaza con un grito y patadas. Con la finalidad de que la niña coma algo, finalmente le dan una pieza de pan con mermelada de fresa, solo le gusta comer pan y mermelada. Esto sucede casi a diario, unas veces en mayor medida que en otras. ¿Podría decirse que Sofía es una niña "berrinchuda" o agresiva? Probablemente sí. La breve descripción del comportamiento de la niña a la hora de comer podría encajar perfectamente en lo que coloquialmente se suele definir como "grosera" o tal vez "niña mala". Ahora, considérese un poco más de información sobre el caso. Cuando Sofía está en casa de su tía, siempre se come lo que ella le prepara de comer, incluso alimentos que ella ha rechazado anteriormente a sus padres ¿Significa esto que Sofía ya no es agresiva? ¿Qué ha cambiado? ¿Es correcto afirmar que Sofía es agresiva sólo a veces? Es importante analizar la diferencia que existe entre la afirmación de "Sofía es agresiva" y la de "Sofía emitió una conducta agresiva". Las implicaciones sociales pueden tener importantes alcances que discutiremos con base en dos enfoques: la perspectiva conductual y la perspectiva incluyente.

En su texto ¿Qué es conducta?, Freixa (2003) expuso algunos malentendidos referentes a la conceptualización de la conducta y el quehacer de la psicología misma. Mediante el uso de metáforas, explicó las confusiones de las que se puede ser presa cuando se habla de conducta, y afirmó que un problema fundamental consiste en situar la conducta 
en el organismo, como si fuese una propiedad de éste. Las repercusiones sociales están relacionadas con la estigmatización y los prejuicios que socialmente ocurren cuando se impone una etiqueta. Así, cuando por ejemplo empieza a hacerse referencia a Sofia como "la niña agresiva" pronto otros familiares comenzarán a describirla de este modo y luego también sus profesores y pares en la escuela.

Cuando se etiqueta a un niño o niña de esta forma, puede ocurrir que se le permita emitir más conductas agresivas que a sus pares pues se pensará "ella es así, es agresiva"; cuando otra profesora pregunte ¿por qué Sofía está golpeando a sus compañeritos? La respuesta será "es que ella es agresiva". Siendo conocida así en su contexto, se esperará que emita este tipo de conductas; esto puede desencadenar la falta de oportunidades de sano desarrollo para esta niña y para todos aquellos que son constantemente etiquetados bajo calificativos como flojo, tonto, llorón, entre otros. Esta supuesta explicación de la conducta que se refiere como problemática es limitada. La causa de la conducta está en la misma conducta, una conceptualización tradicionalmente aceptada, por omisión o conscientemente, por la mayoría de los psicólogos, aunque hay explicaciones más refinadas, metáforas más sofisticadas (Burgos y Killen, 2018).

Ahora bien, desde la perspectiva incluyente, que día a día toma mayor relevancia en el plano sociopolítico, se ha impulsado el uso de un lenguaje en el que prevalece la persona por encima de cualquier característica o condición. Tomemos por ejemplo a un niño diagnosticado con trastorno de déficit de atención con hiperactividad. Es un niño que se distrae fácilmente, no completa las tareas que se le indican, entre otras características. Bajo una perspectiva incluyente se evitaría a toda costa referirse a él como el niño hiperactivo (como desafortunadamente sucede en muchos contextos). Referirse a él por su nombre evitará que se le etiquete y que por tanto sea objeto de prejuicios que afecten sus interacciones sociales. El mismo principio debería aplicar para cualquier otro problema de comportamiento o alteración del desarrollo. Esto no debe ser entendido como un mal intento de negar la existencia de un problema; es más bien que una visión patológica, frecuentemente, impide otro tipo de mirada más optimista terapéuticamente hablando.

Quizá la perspectiva que ofrece más posibilidades a los individuos, a pesar de cualquier pronóstico desalentador, es el Análisis Conductual Aplicado, una práctica basada en la evidencia que ha venido tomando mayor importancia en el terreno clínico gracias a la validez social de sus intervenciones. Es un abordaje científico de las leyes que gobiernan las acciones de los seres humanos y se centra en las conductas observables con relevancia social e importancia clínica mediante su estudio en los escenarios de aplicación (Martin y Pear, 2008). Cooper et al (2020) resaltaron la característica optimista de este enfoque cuando explicaron que para cada individuo hay una posibilidad de mejora. 
La importancia de este tipo de intervenciones exitosas radica justamente en que cuando las personas observan las mejorías conductuales y éstas son clínicamente significativas, su experiencia se modifica: la creencia de que poco o nada se puede hacer para cambiar los problemas conductuales se sustituye por la experiencia exitosa que impacta directamente en la calidad de vida de los sujetos, sus familias y su entorno cercano. Así se forma una nueva construcción social de los problemas conductuales.

\subsection{La afectación bidireccional}

La evidencia es basta con respecto a las repercusiones que tienen las conductas disruptivas sobre el desarrollo de un niño hacia su juventud y su vida adulta. Pero hay evidencia de que dichas dificultades conductuales también repercuten de manera negativa sobre las personas que interactúan con los niños que las presentan. Se han reportado niveles elevados de estrés y burnout en los profesores de niños con problemas conductuales (Friedman, 1995; Hastings y Bham, 2003) y puede afectar no solo a esta población, sino también a los propios padres de familia, personal de cuidado, familiares y en general a la sociedad en sus diferentes formas estructurales y funcionales.

Diversos autores coinciden en señalar que el personal educativo, durante su formación profesional, carecen de las habilidades necesarias para interactuar de manera efectiva con las conductas disruptivas de los niños por lo que pueden percibir que no tienen alternativas más que el uso de intervenciones restrictivas (como inmovilización) especialmente cuando se encuentran frente a conductas agresivas (por ejemplo, golpear o morder a otros) (Knotter et al. 2018; Stormont et al. 2015). Así, además de generar en los niños sentimientos de inseguridad, frustración, enojo, estrés y ansiedad, los problemas conductuales también pueden generar experiencias negativas en los docentes.

Benjet (2015) mostró que en México faltan programas que tengan la finalidad de desarrollar habilidades en los maestros para enfrentar los problemas de comportamiento de los niños con base empírica, sin uso de violencia o de métodos restrictivos, que no generen experiencias negativas en los niños o en quienes les cuidan. Con fundamento en los artículos 57, 58 y 59 de la Ley General de los Derechos de Niñas, Niños y Adolescentes (2014) y en el artículo 11, del capítulo III Condiciones Generales del Servicio del Reglamento de la Ley que Regula a los Centros de Atención Infantil en el Estado de Jalisco (2014), relativos a garantizar los derechos de la infancia, es necesario implementar y evaluar intervenciones conductuales en espacios educativos. En tanto no se implementen acciones en el plano educativo, el problema persistirá.

Sería un error responsabilizar al personal educativo de los problemas de conducta de los niños dado que son muchas las variables involucradas en el comportamiento. Quien haya tenido la oportunidad de interactuar cercanamente con personal educativo, seguramente 
habrá notado que muchos de ellos suelen emitir un conjunto de conductas que podríamos clasificar como "tener pasión por su trabajo" o "tener vocación". No obstante, en el ámbito cotidiano es común reforzar en los niños conductas no deseadas, aún sin la intención de hacerlo (Martin y Pear, 2008). Entonces, sin desearlo, se puede influir en el inicio, mantenimiento y eliminación de una determinada conducta. Técnicamente el reforzamiento adventicio de la conducta socialmente deseada o socialmente no deseable es la consecuencia casi lógica de un sistema educativo que no es ajeno a las prácticas que permean las instituciones burocratizadas $y$, por tanto, que padece los mismos efectos adversos de lo que un sentido bidireccional afecta a la sociedad y a los individuos.

La evidencia al respecto no es reciente, Thomas et al. (1968) evaluaron el efecto de la conducta de una profesora sobre las conductas disruptivas de sus estudiantes. Variaron sistemáticamente las conductas aprobatorias de la profesora (elogio, sonrisa, contacto) y las no aprobatorias (reprimendas verbales y restricción física). Los resultados mostraron que la conducta de aprobación funcionó como reforzador positivo en el mantenimiento de conductas apropiadas en el aula y que los problemas de comportamiento aumentaban cada vez que la maestra las reforzaba con conductas aprobatorias. Los autores indicaron que los maestros no son siempre conscientes (reforzamiento adventicio) de los efectos que tienen sus acciones sobre la conducta de los estudiantes, muchas veces las conductas disruptivas están dentro de su control y pueden modificarlas cuando el maestro controla su propia conducta. Una conclusión lógica del estudio de Thomas et al es que el reforzamiento accidental de conductas no deseadas es consecuencia de la falta de instrucción para el reforzamiento y no la ausencia de éste como frecuentemente se cree y la experiencia clínica así lo demuestra. Los padres, maestros, cuidadores y responsables de la crianza en niños requieren apoyo para modificar el continuo conductual en el momento en el que la probabilidad del cambio es mayor, no así para la disponibilidad de los reforzadores con los que se cuenta para aumentar esta probabilidad.

Podríamos extendernos explicando los fundamentos y los mecanismos por los cuales sucede lo anterior; pero, con la intención de recurrir a las vivencias del propio lector, utilizaremos un ejemplo bastante práctico de Malott et al. (2003) que probablemente resultará familiar para aquellos que han convivido con niños pequeños o con sus cuidadores. Unos padres se encuentran por la noche, cansados, desvelados, tratando de dormir a su bebé, para poder descansar ellos también. Digamos que el bebé está llorando, cuando su madre lo carga en brazos, el bebé comienza a tranquilizarse y eventualmente deja de llorar; la madre, desde luego, se siente aliviada porque el llanto paró. Con el bebé tranquilo, la madre decide que es hora de ponerlo en su cuna, lo hace y sale de puntillas para ir a dormir, pero justo en este momento el bebé comienza a llorar nuevamente. La madre vuelve al cuarto para calmar al bebé como lo hizo anteriormente y sucede lo mismo durante tres horas. Malott et al. (2003) 
llaman a esto ciclo social disfuncional. En este ciclo, hay dos conductas que están siendo reforzadas, seguramente sin la intención de hacerlo. Por un lado, el llanto del bebé es reforzado puesto que va seguido de ser cargado por su madre y, por el otro lado, el acto de cargar al bebé es reforzado ya que, al hacerlo, el bebé deja de llorar. Visto de esta manera podemos comenzar a analizar cuáles son los comportamientos que podemos estar reforzando en otras personas día con día, asimismo, podremos reflexionar sobre la forma en que nuestra propia conducta es reforzada por ciertos estímulos. Esto puede representar un comienzo para comprender por qué hacemos lo que hacemos todos los días, como saludar a nuestros vecinos o ir a trabajar.

\subsection{La propuesta ante este problema en situación de contingencia por COVID- 19}

La pandemia causada por el virus COVID-19 ha pronunciado indudablemente una variedad de problemáticas sociales, económicas, políticas y de salud. Casi todas las personas se han visto obligadas a modificar su forma de relacionarse y su forma de vivir en general. Con el cierre de instituciones educativas, centros de atención infantil y clínicas de atención especializada, los niños, niñas y adolescentes ahora pasan todo o casi todo el tiempo en casa; los cuidadores ya no son los profesionistas especializados sino madres y padres de familia, hermanos y otros familiares cercanos. Los familiares de niños con problemas de conducta han tenido que enfrentar la situación en casa sin estar capacitados para hacerlo. Lo peor, las instituciones a las que pueden recurrir para solicitar apoyo están cerradas o en casos como en México, ni siquiera existen.

Previamente se expuso que las personas suelen buscar ayuda profesional hasta que el problema es más grave o afecta a más personas, es decir, rara vez se atiende desde que se detecta. Si las personas están conviviendo más tiempo debido a la pandemia por COVID19 ¿podría esto contribuir a la búsqueda de ayuda antes de lo usual? Seguramente sí. Foros de discusión y conversaciones informales reflejan preocupaciones generalizadas por exacerbaciones conductuales (completamente esperadas) en población infantil. Las personas parecen buscar la ayuda de expertos más que antes de la pandemia; este acto debe ser alentado dado que siempre es deseable la búsqueda de ayuda cuando se detecta alguna situación problemática y movilizarse al respecto puede verse como un reflejo de cambio en la idea que se tenía del problema.

Los analistas de conducta de diversas partes del mundo han desarrollado estrategias que facilitan el acceso a intervenciones conductuales o asesorías de manera remota mediante el uso de tecnología. La atención a distancia es relativamente reciente, pero ha crecido aceleradamente en el último año y se ha generado evidencia que sugiere que las 
intervenciones a distancia son efectivas tanto para tratar problemas conductuales en los niños como para entrenar a los cuidadores en la atención y manejo de dichos problemas.

Tomlinson et al. (2018) analizaron 20 estudios como parte de una revisión sistemática para sintetizar la literatura concerniente a entrenamientos en procedimientos del análisis conductual aplicado mediante el uso de tecnología a distancia. Participaron profesores, padres de familia y terapeutas que fueron entrenados en un procedimiento conductual específico (evaluación funcional, evaluación de preferencias, ensayos discretos, entrenamiento en comunicación funcional). En general, los estudios reportaron un aumento en las variables observadas.

De manera similar, otros autores (LaBrot et al. 2018; Wilczynski et al. 2017) han evaluado intervenciones en las que cuidadores reciben entrenamiento remoto para mejorar los comportamientos problemáticos de niños bajo su cuidado. Sus resultados proveen evidencia empírica de la utilidad de estos entrenamientos para proveer tratamientos con base analítico conductual. Cabe señalar que estos estudios incorporan elementos tecnológicos para que la presencialidad no sea una limitante; datos que abonan evidencia práctica útil para el contexto por confinamiento.

Como estos estudios hay numerosos intentos recientes por responder a las problemáticas conductuales que se viven actualmente. Tal vez esto represente una oportunidad para que, gracias al uso de la tecnología, el conocimiento llegue a personas a las que no pudiera llegar de otra manera. Quizás este es el momento preciso e idóneo para difundir conocimiento que aporte a desvanecer los estigmas arraigados en nuestra sociedad hacia las cuestiones comportamentales.

Hoy en día se cuenta con protocolos que permiten guiar de manera ética y profesional el actuar de los profesionales en esta materia, tomando en cuenta que las intervenciones a distancia han tomado mayor auge (Council of Autism Service Providers, 2020). Bajo circunstancias de distanciamiento social el reto de seguir atendiendo los problemas de conducta es mayor; la calidad de vida de las personas no puede desatenderse.

\section{Conclusiones}

La construcción social de los problemas conductuales infantiles está influenciada por numerosos elementos. Cada lugar y cada momento tendrá su propia visión sobre el significado de los comportamientos. Si bien se pueden establecer ciertos estándares sobre lo que es normal y lo que no lo es, es importante comprender que lo que para algunas personas representa un problema, para otras no. 
En el presente ensayo se abordaron algunos elementos que influyen en la construcción social de los problemas conductuales; no basta con brindar información si no que las cuestiones estructurales como la disponibilidad de recursos, también influyen en las posibilidades de tratamiento y, por tanto, en la iniciativa para movilizarse al respecto o, por el contrario, percibir que nada se puede hacer y acostumbrarse a eso. Estas experiencias pueden ser modificadas al diseminar los resultados de intervenciones exitosas.

En las últimas décadas, países desarrollados como Estados Unidos han impulsado fuertemente políticas educativas encaminadas a incluir a niños y niñas con o sin discapacidad dentro de los entornos escolares. En México, a pesar de que desde hace tiempo se ha abogado por instalar políticas educativas al respecto, aún no ha tenido el peso que la situación demanda. Se necesitan mayores habilidades en el manejo conductual por parte de los cuidadores.

En los últimos años se ha puesto mayor atención en prácticas basadas en la evidencia, como lo es el Análisis Conductual Aplicado pues es una herramienta efectiva para ayudar a los niños con problemas conductuales a incrementar sus probabilidades de éxito. Este enfoque de modificación de conducta cuenta con principios y métodos claramente definidos que han sido puestos en práctica con éxito en disminución de conductas disruptivas.

Este enfoque de intervención es lo suficientemente sistemático y tecnológico además de fundamentarse en una mirada flexible, que le permite adaptarse a diferentes casos y entornos, sin perder su rigurosidad. Como ya se expuso, su naturaleza optimista es una solución a muchos casos que dejan de ser atendidos y es una excelente alternativa si lo que se busca es desvanecer los estigmas sociales hacia los problemas conductuales.

Es importante dirigir esfuerzos desde el ámbito de la investigación, la formación de recursos humanos y la aplicación con la finalidad de que los países en vías de desarrollo también puedan beneficiarse de este tipo de tecnologías y así contribuir con la calidad de vida de sus habitantes. Pero para ello se requiere que las familias experimenten de primera mano los beneficios comportamentales que se logran con las intervenciones y sepan que en los primeros años se puede mejorar el pronóstico desalentador de un niño.

Finalmente, es necesario enfatizar que un modelo educativo basado en la evidencia práctica y científica puede mejorar la condición de vida no solo de los usuarios sino de los docentes que constituye hasta el día de hoy, uno de los temas pendientes para el sistema educativo nacional. Una deuda que está más allá de lo que una reforma educativa puede ofrecer y que nos involucra a todos como una sociedad que va construyendo y modificando sus propias concepciones sobre los comportamientos problemáticos. 
La primera autora agradece la beca de manutención para estudios de doctorado otorgada por el Consejo Nacional de Ciencia y Tecnología (CONACyT).

\section{Referencias bibliográficas}

Benjet, C. (2015). La salud mental del niño y del adolescente. En M. Medina-Mora, E. Sarti y T. Real (Eds.), La depresión y otros trastornos psiquiátricos (pp. 91-100). Academia Nacional de Medicina,

Benjet, C., Borges, G. y Medina-Mora, M. (2010). Chronic childhood adversity and onset of psychopathology during three life stages: Childhood, adolescence and adulthood. Journal of Psychiatric Research, 44, 732-740.

Boisjoli, R., Vitaro, F., Lacourse, E., Barker, E. y Tremblay, R. (2007). Impact and clinical significance of a preventive intervention for disruptive boys: 15-year follow-up. British Journal of Psychiatry, 191, 415-419. https://doi: 10.1192/bjp.bp.106.030007

Burgos, J. y Killen, P. (2019). Suing for Peace in the War Against Mentalism. Perspectives on Behavior Science, 42, 241-266. https://doi.org/10.1007/s40614-018-0169-2

Cooper, J., Heron, T. y Heward, W. (2020). Applied Behavior Analysis. (3 ed.). Pearson. De la Peña-Olvera, F. y Palacios-Cruz, L. (2011). Trastornos de la conducta disruptiva en la infancia y la adolescencia: diagnóstico y tratamiento. Salud Mental, 34(5), 421427.

Fischer, A., Lehman, E., Miller, J., Houlihan, D., Yamashita, M., O’Neill, R. y Jenson, W. (2019). Integrating school psychology and applied behavior analysis: a proposed training model. Contemporary School Psychology, 23(1), 1-15.

Fombonne, E., Marcin, C., Manero, A., Bruno, R., Diaz, C., Villalobos, M., Ramsay, K. y Nealy, B. (2016). Prevalence of autism spectrum disorders in Guanajuato, México: the leon survey. Journal of Autism and Developmental Disorders, 46, 1669-1685.

Freixa, E. (2003). ¿Qué es conducta? Revista Internacional de Psicología Clínica y de la Salud, 3(3), 595-613.

Friedman, I. (1995). Student behavior patterns contributing to teacher burnout. Journal of Educational Research, 88(5), 281-289. 
Hagan-Burke, S., Kwok, O., Zou, Y., Johnson, C., Simmons, D. y Coyne, M. (2011). An examination of problem behaviors and reading outcomes in kindergarten students. The Journal of Special Education, 45(3), 131-148. https://doi: $10.1177 / 0022466909359425$

Hastings, R. y Bham, M. (2003). The Relationship between Student Behaviour Patterns and Teacher Burnout. School Psychology International, 24(1), 115-127.

Hodgings, S., Larm, P., Ellenbogen, M., Vitaro, F. y Tremblay, R. (2013). Teacher's ratings of childhood behaviors predict adolescent and adult crime among 3016 males and females. The Canadian Journal of Psychiatry, 58(3), 143-150, http://10.1177/070674371305800304.

Knotter, M., Spruit, A., De Swart, J., Wissink, I., Monnen, X. y Stams, G. (2018). Trainigng direct care staff working with persons with intellectual disabilities and challenging behaviour: A meta-analytic review study. Aggression and Violent Behavior, 40, 6072.

LaBrot, Z., Dufrene, B., Whipple, H., McCargo, M. y Pasquea, J. (2018). Targeted and Intensive Consultation for Increasing Head Start and Elementary Teachers' Behavior-Specifc Praise. Journal of Behavioral Education, 29, 717-740.

Malott, W., Malott, E. y Trojan, A. (2003). Principios elementales del comportamiento (4 ed.). (Trad. Pecina, J.). Pearson Educación de México, S.A. de C.V.

Martin, G. y Pear, J. (2008). Modificación de la conducta: qué es y cómo aplicarla (8ed.). (Trad. Vivas, L., \& Suengas, A.). Pearson Educación.

Stormont, M., Reinke, W., Newcomer, L., Marchese, D. y Lewis, C. (2015). Coaching teachers' use of social behavior interventions to improve a children's outcomes: a review of the literature. Journal of Positive Behavior Interventions, 17(2) 69-82.

Thomas, D., Becker, W. y Armstrong, M. (1968). Production and elimination of disruptive classroom behavior by systematically varying teacher's behavior. Journal of Applied Behavior Analysis, 1(1), 35-45.

Tomlinson, S. R. L., Gore, N. y McGill, P. (2018). Training individuals to implement applied behavior analytic procedures via telehealth: a systematic review of the literature. Journal of Behavioral Education, 27, 172-222.

Tonon, G. (2007). Investigar la calidad de vida en Argentina. Psicodebate, 8, 141-150. https://doi.org/10.18682/pd.v8i0.422 
Tremblay, R. (2004). Decade of behavior distinguished lecture: development of physical aggression during infancy. Infant Mental Health Journal, 25(5), 399-407. https://doi: 10.1002/imhj.20015

Tremblay, R. y Côté, S. (2019). Sex differences in the development of physical aggression: An intergenerational perspective and implications for preventive interventions. Infant Mental Health Journal, 40, 129-140. https://doi: 10.1002/imhj.21760.

Valencia, G. y Andrade, P. (2005). Validez del Youth Self Report para problemas de conducta en niños mexicanos. International Journal of Clinical and Health Psychology, 5(3), 499-520.

Vitaro, F., Tremblay, R., Brendgen, M. y Larose, S. (2005). Kindergarten disruptive behaviors, protective factors, and educational achievement by early adulthood. Journal of Educational psychology, 97(4), 617-629. https://doi: 10.1037/0022-0663.97.4.617

Wilczynski, S., Labrie, A., Baloski, A., Kaake, A., Marchi, N. y Zoder-Martell, K. (2017). Webbased teacher training and coaching/feedback: a case study. Psychology in the Schools, 54(4), 433-445.

Zavaleta, P., Cordero, M., Hernández, A y Medina, M. (2018). Infraestructura disponible para la atención de los trastornos del espectro autista en el sistema nacional de salud. Instituto Nacional de Psiquiatría Ramón de la Fuente Muñiz, Secretaría de Salud, México.

\section{Otros documentos consultados}

Congreso General de los Estados Unidos Mexicanos (4 de diciembre de 2014). Ley General de los Derechos de Niñas, Niños y Adolescentes. Diario Oficial de la Federación. http://www.dof.gob.mx/nota_detalle.php?codigo=5374143\&fecha=04/12/2014

Council of Autism Service Providers (2020). Practice Parameters for TelehealthImplementation of Applied Behavior Analysis: Continuity of Care during COVID-19 Pandemic. Wakefield.

Gobierno Constitucional del Estado Libre y Soberano de Jalisco (2014). Reglamento de la Ley que Regula a los Centros de Atención infantil en el Estado de Jalisco.

Organización de las Naciones Unidas para la Educación, la Ciencia y la Cultura (UNESCO) (2020). https://es.unesco.org/ 